Report NPI Řež-TH-03/2000

\title{
On the reliability of the theoretical internal conversion coefficients*
}

\author{
M.Ryšavý† O. Dragoun \\ Nuclear Physics Institute, Acad. Sci. of Czech Republic, \\ CZ-250 68 Řež near Prague, Czech Republic
}

\begin{abstract}
Possible sources of uncertainties in the calculations of the internal conversion coefficients are studied. The uncertainties induced by them are estimated.
\end{abstract}

PACS: $23.20 . \mathrm{Nx}$

\section{Introduction}

The internal conversion coefficients (ICC) serve many years as an indispensable tool to assign spin-parity quantum numbers of excited nuclear levels. There exist widely distributed tables [1, 2, 3] of ICC covering broad region of transition energies, atomic numbers from $Z=30$ [1, 3] or 10 [2] up to $Z=103$ [3] or $Z=104$ [1], 2] and transition multipolarities M1 - M4, E1 - E4. Very recently, tables of ICC for the superheavy elements $(104 \leq \mathrm{Z} \leq 126)$ appeared [1].

When computing ICC, however, various approximations are applied. Some of them are of purely theoretical origin, e.g. the calculations are done only for the first nonvanishing order of the perturbation theory. Other ones are connected with improper description of physical reality, e.g. the ICC is calculated for a free atom whereas the real source atom is usually chemically bound in some compound or embedded into a solid. In this work we systematize these particular sources of uncertainties and estimate their effect on the accuracy of the calculated ICC.

\footnotetext{
${ }^{*}$ Main part of this paper was presented at the meeting of Decay Data Evaluation Project, Braunschweig, May 2000

†e-mail: rysavy@ujf.cas.cz
} 


\section{Basic formulae}

The basic formulae for the ICC together with its derivation may be found in many works. We present here those from the monography [5]. The ICC for the transition of the multipolarity $\tau L$ and the atomic shell $i$ is given by

$$
\alpha_{i}(\tau L)=\alpha \pi \omega \frac{2 j_{i}+1}{L(L+1)} \sum_{\kappa_{f}}\left|C_{j_{i},-1 / 2, L, 0}^{j_{f},-1 / 2}\left(\mathcal{R}_{\kappa_{f}}^{(\tau L)}+\mathcal{T}_{\kappa_{f}}^{(\tau L)}\right)\right|^{2} .
$$

Here

$$
\begin{array}{r}
\mathcal{R}_{\kappa}^{(M L)}=\int_{0}^{\infty} \mathcal{F}_{\kappa}^{(0)}(r) h_{L}(\omega r) d r \\
\mathcal{R}_{\kappa}^{(E L)}=\int_{0}^{\infty}\left[L \mathcal{F}_{\kappa}(r) h_{L}(\omega r)+\mathcal{F}_{\kappa}^{(-1)}(r) h_{L-1}(\omega r)\right] d r .
\end{array}
$$

and

$$
\begin{array}{r}
\mathcal{F}_{\kappa_{f}}^{(0)}(r)=\left(\kappa_{i}+\kappa_{f}\right)\left(u_{\kappa_{i}} v_{\kappa_{f}}+u_{\kappa_{f}} v_{\kappa_{i}}\right), \\
\mathcal{F}_{\kappa_{f}}(r)=\left(u_{\kappa_{i}} u_{\kappa_{f}}+v_{\kappa_{i}} v_{\kappa_{f}}\right), \\
\mathcal{F}_{\kappa_{f}}^{(-1)}(r)=\left(\kappa_{i}-\kappa_{f}-L\right) v_{\kappa_{i}} u_{\kappa_{f}}+\left(\kappa_{i}-\kappa_{f}+L\right) u_{\kappa_{i}} v_{\kappa_{f}} .
\end{array}
$$

$C_{j_{1}, m_{1}, j_{2}, m_{2}}^{j, m}$ is Clebsch-Gordan coefficient, $\alpha$ is the fine structure constant, $h_{l}(x)$ is the spherical Hankel function, $\omega$ is transition energy, $u_{\kappa}$ and $v_{\kappa}$ are small and great, respectively, component of the solution of Dirac equation for electron.

For the transition of magnetic multipolarity, the quantity

$$
\mathcal{T}_{\kappa_{f}}^{(M L)}=\frac{\int d^{3} R \vec{J}_{\text {nucl }}(\vec{R}) \hat{\vec{L}} g_{\kappa_{f}}^{(3)}(R) Y_{L M}^{*}}{\int d^{3} R \vec{J}_{\text {nucl }}(\vec{R}) \hat{\vec{L}} j_{L}(\omega R) Y_{L M}^{*}}
$$

where

$$
g_{\kappa}^{(3)}(R)=h_{L}(\omega R) \int_{0}^{R} d r j_{L}(\omega r) \mathcal{F}_{\kappa}(r)-j_{L}(\omega R) \int_{0}^{R} d r h_{L}(\omega r) \mathcal{F}_{\kappa}(r)
$$

is the only one which containes nuclear variables. It describes "intranuclear conversion" (see Sect. 3.7). For the electric transitions, the analogical quantity $\mathcal{T}_{\kappa_{f}}^{(E L)}$ is given by a more complicated formula but it has similar structure.

From the formulae above we see that the ICC depends on four 'parameters': transition energy $\omega$, transition multipolarity $\tau L$, initial electron state (i.e. atomic shell), and atomic number $Z$. For the atomic number, the dependence is purely implicit - via atomic potential which then forms the electron wave functions $u_{\kappa}$ and $v_{\kappa}$. The other quantities enter into the formulae explicitly but, moreover, they also affect the result indirectly. E.g. the transition multipolarity determines the allowed final electron states due to the conservation of angular momentum. It is obvious that to draw some information on the shape of the dependencies directly from the above formulae is almost impossible. The only way is to evaluate ICC for various parameters and find out the dependencies from the numerical results. Analogically, the effect of various approximations and/or model imprefections may also be studied via numerical calculations. 


\section{Sources of the ICC uncertainties}

When we put a question about the precision of the theoretical ICC we must first specify the possible sources of their uncertainties. These may be very roughly divided into two classes - those stemming from theory and those given by our insufficient knowledge of some quantities entering the calculations. Into the first category there can be included atomic model, nuclear deformation and nonsphericity in general, and higher order effects. The second one consists of atomic binding energies, isotopic effect, and chemical effects. Somewhere in-between is the question of correct accounting of nuclear structure, i.e. the intranuclear conversion. And, finally, a serious error may be introduced by incorrect interpolation in existing tables, especially in case of total ICC.

In the following, we address ourselves to the particular items listed above. We summarize all the known facts and try to give a realistic estimate of the uncertainties induced.

\subsection{Atomic model}

As seen from Eqs. (1 - 6), the electron wave functions $\left(u_{\kappa}\right.$ and $\left.v_{\kappa}\right)$ are needed to calculate an ICC. The evaluation of these wave functions is based on some suitable model of atom.

When we skip the 'ancient' calculations with the model of point nucleus without screening, the first more realistic ICC values for the $\mathrm{K}$ and L shells were obtained [6]- [8] using the Thomas-Fermi-Dirac atomic model. Later on this model was abandoned in favour to physically more founded model of Hartree and Fock with the Slater exchange term (HFS). This atomic model was employed to provide the first tables of the N-shell ICC [9]. A little sooner in Ref. [3], a modified HFS method where the Slater exchange term is weighted by a factor of $2 / 3$ (so called $\mathrm{HF} \frac{2}{3} \mathrm{~S}$ method) was utilized to get the ICC for the shells $\mathrm{K}$, L, and M.

In the late 70', the most extensive tables [1, 2] appeared where the pure HFS model was applied. Approximately at the same time the first ICC based on the true Hartree-Fock (HF) model (without the Slater exchange term) were calculated [10, 11]. To complicate the things yet more, it is not clear whether the 'hole' in the atomic shell remaining after the conversion electron should be taken into account or not.

Evidently one should hesitate which ICC are the "real" ones and what error can be made by using other ones. Band et al. [12] performed extensive calculations of ICC in both models HFS and $\mathrm{HF} \frac{2}{3} \mathrm{~S}$ and for both variants 'hole' and 'no hole'. They evaluated large sets of ICC for $Z=30,60,90$, conversion electron energy between 10 and $400 \mathrm{keV}$, multipolarities M1 - M4 and E1 - E4, and $\mathrm{K}, \mathrm{L}_{1}, \mathrm{~L}_{2}$ and $\mathrm{L}_{3}$ subshells. Then they constructed the quantities which may characterize the uncertainty caused by the atomic model,

$$
\begin{aligned}
\Delta_{1} & =\left[\frac{I C C(\text { hole })}{I C C(\text { no hole })}-1\right] \times 100, \\
\Delta_{2} & =\left[\frac{I C C\left(H F \frac{2}{3} S\right)}{I C C(\text { HF })}-1\right] \times 100,
\end{aligned}
$$

where the symbol in parentheses indicates the model used. These quantities vary with multipolarity - in general, their absolute values increase with $L$. They increase with 
decreasing energy. For some combinations of subshell and multipolarity they do not exceed $0.5 \%$, for the remaining ones there are graphs in [12].

In [11], an extensive comparison of HF model[ ICC with experimental ones was performed. Altogether 57 experimental data items for 8 transitions in six different isotopes were examined. A sensitive statistical test revealed better agreement of the theory with experiment for the HF ICC than for the HFS ones. The differences between the two sets of theoretical ICC varied from $\lesssim 1 \%$ on the K-shell, $2-3 \%$ on the L-subshells up to 30\% on the outermost shell.

More recently, there appeared [14] the 'true' HF model ICC, i.e. those where even the free-electron wave functions were evaluated without the approximation of the exchange term. 15 transitions in various isotopes were studied and better agreement of the theory and experiment was confirmed.

Tables of ICC in the HF model are not available at present. However, as stated above, programs exist and it is possible to calculate the HF (i.e. physically best) ICC for particular cases. As for their uncertainty, only that corresponding to numerical accuracy (mostly $<0.5 \%$ ) may be expected. For the other atomic models, it is difficult to give some general error estimate. It might be the difference from the HF ICC. Anyway these quantities are known for several particular transitions only (e.g. [11, 14]). And in any case, such an 'error' would be a systematic rather than statistical one.

Recommendation: For analysis of precise experimental data, use the HF model based ICC.

\subsection{Nuclear deformation and nonsphericity}

The formulae for conversion coefficients are based on the assumption that the atomic field is spherically symmetrical.

It has been proved long time ago [15] that the closed shells form a symetrical atomic field. With several exceptions ( $\llbracket 16]$, p. 74), there is only one unclosed shell in every free atom. This is the valence shell which, in most cases, is the outermost one. (Even if there are two unclosed shells, they always belong to the outer ones.) The calculated ICC is in fact the mean over all projections of the angular momentum. This evidently brings an error in the case of unclosed shells. If the actual occupation number for the particular shell, $i$, is $n_{a c t}$ and the maximum possible one is $n_{0}$, the conversion coefficient should be rescaled as

$$
\alpha_{i}(a c t)=\frac{n_{a c t}}{n_{0}} \times \alpha_{i}(\text { calc }),
$$

where $\alpha_{i}($ calc $)$ is the calculated ICC. It greatly reduces the error caused by the absence of one or several electrons. The error due to the asymmetry remains but it can be negtected. The asymmetry is in fact a slight perturbation which affects almost exclusively only the particular unclosed shell. As stated above, it concerns the outer shells and there is a greater uncertainty due to the chemical environment (Sect. 3.6).

\footnotetext{
${ }^{1}$ In fact, only the bound electron wavefunctions are calculated in HF model there. The wavefunctions of the emitted electrons are generated as previously is HFS model. It was proved that the free electron wavefunctions are much less sensitive to the model details (e.g. [13).
} 
Another possible source of asymmetry can be a deformed atomic nucleus. The effect of nuclear deformation on the ICC was studied in [17, 18, 19]. The authors [17, 18] assumed a nucleus with high quadrupole deformation and used perturbation series to compute contributions to ICC. Such calculations were done for ${ }_{66}^{160} \mathrm{Dy},{ }_{68}^{166} \mathrm{Er},{ }_{70}^{170} \mathrm{Yb}$, and ${ }_{76}^{186}$ Os nuclei exhibiting high quadrupole moments. The comparison of the results with the ICC evaluated in a usual way (i.e. for spherical nucleus) revealed a negligible change of $\sim 10^{-2} \%$. In [19], also the effect of magnetic dipole moment was investigated and found to be negligible.

It should be kept in mind that the transitions in deformed nuclei are often hindered. In such cases it may be necessary to account for the intranuclear conversion (Sect. 3.7).

Recommendation: If using the conversion coefficient for a subshell which is not completely filled do not forget to rescale it following Eq.(11). If studying a transition in a deformed nucleus, think on possibility of intranuclear conversion.

\subsection{Higher order effects}

At present, all ICC are evaluated up to the first non-vanishing order of perturbation theory, i.e. the first order for the gamma emission and second one for the emision of conversion electron. The Feynman diagrams corresponding to the next nearest orders are presented in Fig.1. The contributions of individual graphs to ICC were estimated by several authors, e.g. [20], 30, [14].

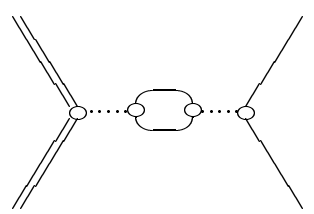

a) vacuum polarization

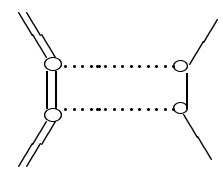

d) nucleus-electron bridge

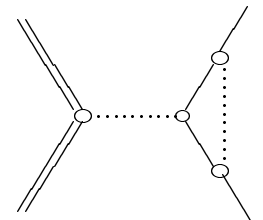

b) vertex correction

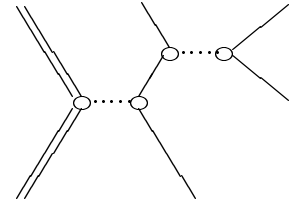

e) electron bridge

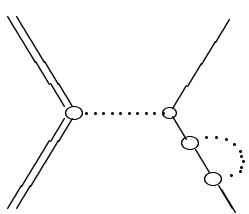

c) self-energy

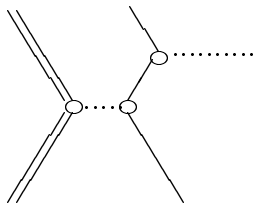

f) photon emission via electron bridge

Figure 1: Feynman diagrams for internal conversion up to the fourth order and for $\gamma$-ray emision up to the third order of perturbation theory.

Vacuum polarization. It is the only higher order effect which can be taken into acount precisely by a change of atomic potential. It was studied in [26]. The authors found small influence on ICC, namely a change of $<0.1 \%$ for $Z=26$ and $<1 \%$ for $Z=80$.

Vertex correction. Contribution of vertex correction to ICC was never investigated. Pauli et al. [25] stated that Hager and Seltzer [24] studied it. However, the graphs 
presented in 24 correspond to 'electron bridge' - graphs (e) and (f) in Fig.1.

Self-energy. Up to our knowledge the possible contribution of this term to the ICC was never examined.

Bridges. These corrections were extensively investigated by Krutov et al. [20]- 23]. Unfortunately, there are almost none numerical estimates. The up to $20 \%$ effect [22] of the graph $(\mathrm{d})$ in one specified case seems unrealistic (during the calculations, a lot of terms which can contribute were neglected). Hager and Seltzer [24] stated about $5 \%$ efect of (e) and (f) on the L-subshell ICC in ${ }_{74}^{182} \mathrm{~W},{ }_{69}^{169} \mathrm{Tm}$, and ${ }_{66}^{160} \mathrm{Dy}$. Also here, several important terms were neglected and, moreover, the Pauli principle was not fulfilled in their calculations. On the other hand, Mayol et al. [27] found the electronic bridge effect of $0.0047 \%$ in ${ }_{77} \mathrm{Ir}$ atom which is stated to be the maximum one from 11 isotopes studied. Hinneburg et al. [28] reported extremely small contribution of $10^{-9}$. In the later works [29, 30] they concentrate to the transition with the energy of $77 \mathrm{eV}$ in ${ }_{92}^{235 m} \mathrm{U}$ where the graph (f) could raise the photon decay constant by a factor of $\sim 10^{5}$ and thus suppress the ICC. Their calculations, however, cannot be verified since the ICC of such a low energy transition is unmeasurable at present. Band et al. [14] admit the effect of 'electron bridge' for very low transition energies but do not present any concrete estimates.

Anyway, the above approach - the evaluation of contributions of individual graphs - is not correct. The right method is first to derive the matrix elements corresponding to particular graphs and add them together. Only then the modified integrals (2), 33, (7) can be applied in the final formula (1). This is such a difficult task that nobody even dared to perform it up to now.

Recommendation: For the transition energies not too close to conversion threshold, assume the uncertainty caused by neglection of higher orders to be about $1 \%$. This estimate follows from analogy with electron Coulomb scattering using the formulae in [31]. As for the very low transition energies, we can say nothing at present.

\subsection{Atomic binding energies}

The binding energies, $E_{b, i}$, of the atomic electrons enter into the ICC via the relation

$$
E_{k i n, i}=E_{t r}-E_{b, i}
$$

where $E_{k i n, i}$ and $E_{t r}$ are kinetic energy of conversion electron and transition energy, respectively. In Eq.(12), the recoil energy as well as work function of the source material are neglected. The $E_{k i n, i}$ specifies the conversion electron wave functions appearing in formulae (14).

The binding energies are presented in [32] with the typical error of $\pm 1 \mathrm{eV}$, sometimes 2 or $3 \mathrm{eV}$. (An exception are several transuranic elements where the stated error amounts in some cases up to $\pm 18 \mathrm{eV}$.) To estimate the uncertainty in ICC caused by those uncertainties in $E_{b, i}$ we performed calculations with various values of binding energies. In particular, we evaluated ICC for ${ }_{26} \mathrm{Fe},{ }_{55} \mathrm{Cs}$ and ${ }_{87} \mathrm{Fr}$ and the subshells $\left(\mathrm{K}, \mathrm{M}_{3}\right),\left(\mathrm{K}, \mathrm{N}_{5}\right)$ and $\left(\mathrm{K}, \mathrm{O}_{5}\right)$, respectively. This setting was chosen to cover low, medium and high $\mathrm{Z}$ and always one inner and one outer shell. The transition energies of 30, 50, 100, 200, 300 and $500 \mathrm{keV}$ were used where applicable. Finally, the ICC were evaluated always for three binding energies, $E_{0}-10 \mathrm{eV}, E_{0}$, and $E_{0}+10 \mathrm{eV}$ where $E_{0}$ is the binding energy [32]. (Note 
that the binding energies - including those for the inner shells - may also be influenced by the chemical state of the converting atom. These so called chemical shifts, however, are rather small and our interval of $20 \mathrm{eV}$ covers them all right.)

Table 1: Internal conversion coefficients for ${ }_{55} \mathrm{Cs}$, shell K, transition energy of $50 \mathrm{keV}$ and three binding energies.

\begin{tabular}{clllr}
\hline mult. & $E_{0^{-}} 10 \mathrm{eV}$ & $E_{0}{ }^{a}$ & $E_{0}+10 \mathrm{eV}$ & $\Delta^{b}$ \\
\hline $\mathrm{E} 1$ & $1.2320(0)^{c}$ & $1.2318(0)$ & $1.2317(0)$ & .02 \\
$\mathrm{E} 2$ & $7.0612(0)$ & $7.0563(0)$ & $7.0513(0)$ & .07 \\
$\mathrm{E} 3$ & $2.6671(1)$ & $2.6632(1)$ & $2.6593(1)$ & .29 \\
$\mathrm{E} 4$ & $9.2835(1)$ & $9.2622(1)$ & $9.2408(1)$ & .46 \\
$\mathrm{M} 1$ & $5.8912(0)$ & $5.8912(0)$ & $5.8911(0)$ & $<.01$ \\
$\mathrm{M} 2$ & $9.4640(1)$ & $9.4617(1)$ & $9.4594(1)$ & .05 \\
$\mathrm{M} 3$ & $6.1914(2)$ & $6.1863(2)$ & $6.1811(2)$ & .17 \\
$\mathrm{M} 4$ & $3.1418(3)$ & $3.1369(3)$ & $3.1320(3)$ & .31 \\
${ }^{a} E_{0}=36189.9 \mathrm{eV}$ & & \\
${ }^{b}$ Maximum change in percent, $\Delta=\left(\frac{\max }{\min }-1\right) \times 100$ \\
${ }^{c} \mathrm{x}(\mathrm{y})$ means $\times 10^{y}$
\end{tabular}

As a measure of the uncertainty we take the quantity $\Delta=\left(\frac{\max }{\min }-1\right) \times 100$ where max and min are the maximum and minimum, respectively, ICC from the three ones corresponding to different binding energies. For the innermost $(\mathrm{K})$ shell, the results are following. In almost all cases, $\Delta$ is of order of $10^{-2}$ of percent, up to $0.1 \%$. The exception is the $50 \mathrm{keV}$ transition in ${ }_{55} \mathrm{Cs}$ (see Tab.11). For high multipolarities, the changes there reach almost $0.5 \%$. The reason is simple - we are closer to threshold than in the other cases. (When approaching the threshold, the kinetic energy decreases and its change plays more important role.) For the outer shells, the changes are even less.

The change of binding energies used here $(20 \mathrm{eV})$ is relatively large. If the experimental error of binding energy is less (which is usually the case), the corresponding uncertainty in ICC is also less.

Recommendation: If the binding energy is known with a precision better than say $\pm 5 \mathrm{eV}$ and the transition energy is high enough (20 keV or more above the threshold), the uncertainties caused by it in ICC are negligible. This is true even when we keep in mind the possible chemical shifts of binding energies. For the transition energy very close to threshold, no general rule can be given and the problem must be solved by calculating ICC for different binding energies.

\subsection{Isotopic effect}

The mass number, $A$, does not enter the formulae for ICC explicitly. Nevertheless it determines the nuclear radius

$$
r_{n u c l}=r_{0} \times A^{1 / 3}
$$


This implies that $A$ affects the atomic potential which determines the electron wave functions. We describe the atomic nucleus by the Fermi distribution of charge

$$
\rho(r)=\frac{\rho_{0}}{1+\exp \left(\frac{c-r}{t}\right)},
$$

where $c$ and $r_{\text {nucl }}$ are tabulated in [33] and $t$ is found from the condition $\rho\left(r_{\text {nucl }}\right)=0.05 \rho_{0}$.

We assume that $r_{n u c l}$ in [33] corresponds to $A$ of the most abundant isotope for every element. To obtain ICC accordant with another mass number, $A^{\prime}$, we rescaled the nuclear radius by the ratio $\left(A^{\prime} / A\right)^{1 / 3}$. The calculations were performed for the same set of elements, transition energies, shells and multipolarities as that in Sect.(3.4). ICC were evaluated for three different mass numbers, $A$ and $A \pm 10$, in ${ }_{55} \mathrm{Cs}(A=133)$ and ${ }_{87} \mathrm{Fr}$ $(A=223)$ and for two mass numbers (57 and 67$)$ in the case of ${ }_{26} \mathrm{Fe}$.

The results are following: In iron and cesium, only very small changes (up to $\sim 0.2 \%$ ) were observed for the K-shell ICC. For the outer subshells considered, the changes were significantly lower. In the francium, the changes turned out to be higher (see Tab.2). Generally, the changes do not depend to transition energy. This indicates that they are

Table 2: Internal conversion coefficients for ${ }_{87} \mathrm{Fr}$, shell $\mathrm{K}$, transition energy of $200 \mathrm{keV}$ and three mass numbers.

\begin{tabular}{clllr}
\hline mult. & $A=213$ & $A=223$ & $A=233$ & $\Delta^{a}$ \\
\hline E1 & $7.0255(-2)^{a}$ & $7.0251(-2)$ & $7.0250(-2)$ & 0.01 \\
E2 & $1.6199(-1)$ & $1.6197(-1)$ & $1.6196(-1)$ & 0.02 \\
E3 & $3.8503(-1)$ & $3.8490(-1)$ & $3.8473(-1)$ & 0.08 \\
E4 & $9.6301(-1)$ & $9.6236(-1)$ & $9.6176(-1)$ & 0.13 \\
M1 & $1.7636(0)$ & $1.7586(0)$ & $1.7538(0)$ & 0.56 \\
M2 & $6.6561(0)$ & $6.6465(0)$ & $6.6379(0)$ & 0.27 \\
M3 & $1.7317(1)$ & $1.7295(1)$ & $1.7277(1)$ & 0.23 \\
M4 & $4.2730(1)$ & $4.2689(1)$ & $4.2642(1)$ & 0.21 \\
\hline$a$ See
\end{tabular}

${ }^{a}$ See comments on Tab.1]

caused mostly by slight alterations of the bound-electron wave functions. As could be expected, the K-shell is affected most of all. Moreover, the magnetic transitions are more sensitive than the electric ones.

Recommendation: In most cases, the uncertainties caused by isotopic effect are negligible. In case that data on some extremely heavy or extremely light isotop of a given element are studied, allow for uncertainty of up to $0.5 \%$ to be on the safe side. A better alternative is to evaluate ICC directly for the given $A$ which eliminates this kind of uncertainties completely.

\subsection{Chemical effects}

The conversion coefficients are always evaluated under the assumption that the source atom is an isolated free neutral atom. In practice, the experimental ICC are obtained from a radioactive source where the radiating atoms are bound in some structure - molecule or crystal. 
There were attempts to account for the source structure. The Wigner-Seitz boundary condition introducing solid state effects was applied in calculations [27]. The ICC were evaluated for 11 transitions in various isotopes. It turned out that the K-shell and total ICC were almost the same as those evaluated in the free atom approximation. (As for the other shells, no information is given in [27].) In [34, the ICC for the free atom were compared with those calculated using the $\mathrm{X} \alpha$-SW description of a Tc compound. The difference between the two total ICC was of order of $10^{-1} \%$. In [35], the X $\alpha$-SW calculations for some tellurium compounds gave electron densities near the Te nucleus. The free-atom ICC were then scaled as ICC $($ real $)=\left|\psi^{X \alpha-M S}\left(r_{\text {nucl }}\right)\right|^{2} /\left|\psi^{\text {free }}\left(r_{\text {nucl }}\right)\right|^{2} \times$ ICC (free), where $|\psi|^{2}$ 's are the bound electron densities at the nuclear edge. Unfortunately this attepmt failed to bring the theoretical ICC to an agreement with experimental ones for several Te compounds.

Another possibility is to approximate the 'real' ICC by their values evaluated for free ions. The atom bound e.g. in a molecule certainly is not a free ion. In spite of this the ICC for free ions were calculated [36]-39] (for ${ }_{52}^{125} \mathrm{Te},{ }_{36}^{83} \mathrm{Kr},{ }_{83}^{206} \mathrm{Bi}$, and ${ }_{59}^{142} \mathrm{Pr}$, respectively) and it turned out that the experimental outer-shell ICC ratios lay within the range of theoretical values for reasonably ionized atoms. This gives us an argument to use the dispersion of ICC calculated for several ionic states as a measure of uncertainty caused by the chemical bond.

First, we performed extensive calculation of ICC in ${ }_{55}^{133} \mathrm{Cs}$ over a broad region of ionizations (from -1 up to +9 ). The multipolarities M1 - M4 and E1 - E4, all atomic shells, and the energies of $30,100,200$, and $500 \mathrm{keV}$ were treated. The method of calculation is described in [37].

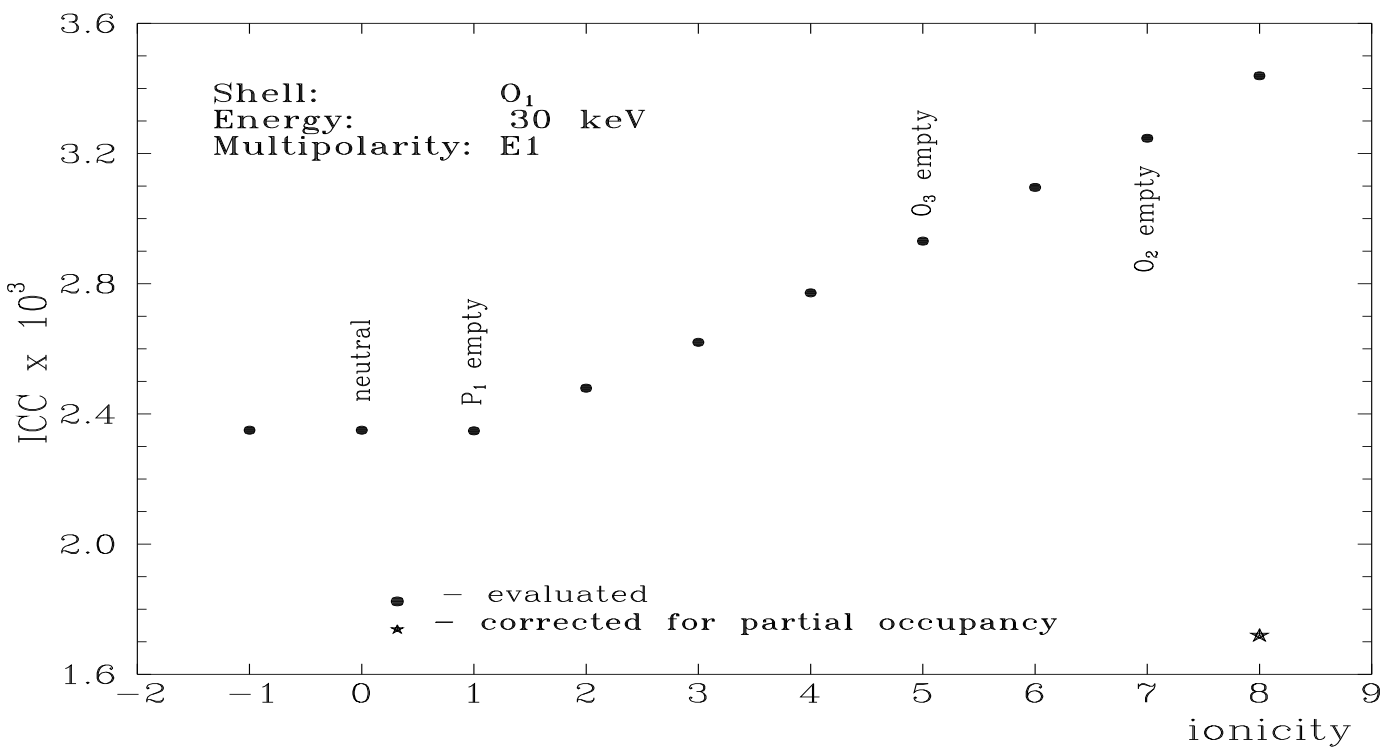

Figure 2: ICC on the $\mathrm{O}_{1}$-subshell of ${ }_{55} \mathrm{Cs}$ in dependence of ionization of the atom. With the exception of the ionization +8 , the $\mathrm{O}_{1}$-subshell is fully occupied.

As a measure of uncertainty we used again the quantity $\Delta$ introduced in Sect 3.4 (Tab.1) but now the maximum and minimum is always taken over all ionizations. The magnitudes of $\Delta$ were as follows: $\lesssim 0.2 \%$ (for the shells $\mathrm{K}, \mathrm{L}_{1}-\mathrm{L}_{3}, \mathrm{M}_{1}-\mathrm{M}_{3}$ ), $\sim 0.3 \%$ 
$\left(\mathrm{M}_{4}, \mathrm{M}_{5}\right), \sim 0.6 \%\left(\mathrm{~N}_{1}\right)$, increasing up to $10 \%$ (for $\mathrm{N}_{5}$ ), and several tens of percent (O-subshells). For the total ICC, $\Delta$ was mostly $\lesssim 0.3 \%$, exceptionally $\sim 0.5 \%$ (for M1 transition, energy $30 \mathrm{keV}$ ). The results are not too dependent on both energy and multipolarity. For illustration there are results for the subshell $\mathrm{M}_{2}$ in Tab.3. The changes of ICC on the $\mathrm{O}_{1}$ shell shows Fig.2.

Table 3: Uncertainties (in percent) of ICC on the $\mathrm{M}_{2}$-subshell of ${ }_{55}^{133} \mathrm{Cs}$ caused by chemical binding.

\begin{tabular}{cllll}
\hline & \multicolumn{4}{l}{$\mathrm{E}_{\gamma}[\mathrm{keV}]$} \\
\cline { 2 - 5 } mult. & 30 & 100 & 200 & 500 \\
\hline E1 & 0.10 & 0.09 & 0.09 & 0.07 \\
E2 & 0.07 & 0.07 & 0.11 & 0.08 \\
E3 & 0.07 & 0.07 & 0.07 & 0.11 \\
E4 & 0.17 & 0.08 & 0.06 & 0.07 \\
M1 & 0.10 & 0.13 & 0.10 & 0.08 \\
M2 & 0.08 & 0.07 & 0.10 & 0.07 \\
M3 & 0.20 & 0.09 & 0.11 & 0.06 \\
M4 & 0.22 & 0.10 & 0.07 & 0.07 \\
\hline
\end{tabular}

To get more information on the 'chemical' uncertainties, we extended our study to more elements. In particular ${ }_{87} \mathrm{Fr}$ - together with ${ }_{55} \mathrm{Cs}$ - belongs to alcaline metals, i.e. those having one electron above the noble-gas core. Then ${ }_{35} \mathrm{Br},{ }_{53} \mathrm{I}$, and ${ }_{85} \mathrm{At}-$ here, one electron lacks to noble-gas-like closed shells. And finaly ${ }_{26} \mathrm{Fe},{ }_{62} \mathrm{Sm}$, and ${ }_{76} \mathrm{Os}-$ the transition metals. This set covers various configurations of the valence shell. Moreover, we added the transition energies of 50 and $300 \mathrm{keV}$ to suppress possibility of some unobserved changes of the uncertainty.

The results confirmed the conclusions made from the case of ${ }_{55} \mathrm{Cs}$. The changes are of tenths of percent for the iner shells and increase to tens of percent for the outermost shell. The changes (uncertainties) of the total ICC are presented in Tab. 4 together with the ranges of ionization used for particular elements. We see that the uncertainty of total

Table 4: Maximum changes of total ICC over all the ionizations given

\begin{tabular}{rrrr}
\hline & \multicolumn{2}{c}{ ionization } & change \\
\cline { 2 - 3 } & from & to & {$[\%]$} \\
\hline${ }_{26} \mathrm{Fe}$ & 0 & +4 & 0.7 \\
${ }_{35} \mathrm{Br}$ & -1 & +4 & 0.5 \\
${ }_{53} \mathrm{I}$ & -1 & +4 & 0.2 \\
${ }_{55} \mathrm{Cs}$ & -1 & +9 & 0.5 \\
${ }_{62} \mathrm{Sm}$ & 0 & +5 & 0.1 \\
${ }_{76} \mathrm{Os}$ & -1 & +5 & 0.1 \\
${ }_{85} \mathrm{At}$ & -1 & +4 & 0.1 \\
${ }_{87} \mathrm{Fr}$ & 0 & +8 & 0.2 \\
\hline
\end{tabular}

ICC due to the chemical effects decreases with increasing atomic number, $Z$. This is not 
surprising - the higher $Z$, the more atomic subshells. And only the lower ones (which are less affected) contribute significantly into the total.

Recommendation: If no information on the structure of the source exists, the quantities presented in Tab.4 may be used as very conservative estimates of the error. On the other hand if some information is available, the present values may be modified (usually decreased) or neglected.

\subsection{Intranuclear conversion}

The intranuclear conversion (also called nuclear structure effect, penetration effect) is responsible for that part of ICC which corresponds to the integral (đ). Physically it is caused by non-zero probability that the electron penetrates into the nucleus and the conversion process takes part there.

In principle, the nuclear currents $\vec{J}_{n u c l}(\vec{R})$ in Eq.(7) may be determined from a suitable nuclear model and the integral then may be evaluated. Such an ICC, however, would be model-dependent. Moreover, the reliability of nuclear models is not sufficient. Therefore, two approximations were introduced. The first one, the Rose's 'no penetration' model [6, 40], completely neglects the contribution (7). The second one, the Sliv's 'surface current' model [41] assumes that the nuclear transition currents are localized at the nuclear surface which enables the evaluation of the integral (7).

Note that the both approaches are approximations only (even when each of them is supported by some physical basement). A convenient way how to manage the contribution of the intranuclear conversion was developed by Pauli [42]. He expressed the ICC involving the contribution (7) as

$$
\begin{array}{r}
\alpha_{i}(M L)=\alpha_{i}^{0}(M L) \times\left(1+b_{1 i} \lambda+b_{2 i} \lambda^{2}\right) \\
\alpha_{i}(E L)=\alpha_{i}^{0}(E L) \times\left(1+a_{1 i} \eta+a_{2 i} \eta^{2}+a_{3 i} \eta \xi+a_{4 i} \xi+a_{5 i} \xi^{2}\right)
\end{array}
$$

where $\alpha_{i}^{0}$ is the ICC on the subshell $i$ calculated in the 'no penetration' model, the formulae for $a$ 's and $b$ 's contain only electronic variables. All nuclear variables from Eq.(7) are constricted into one $(\lambda)$ and two $(\xi, \eta)$ parameters for the ML and EL multipolarities, respectively, which, in turn, contain no electronic variables. These parameters should be determined by comparison of the theoretical and experimental ICC using e.g. the program 433 or, in principle, they may be calculated in frame of a specific nuclear model.

The possible effect, i.e. the magnitude of the polynomials in parentheses in Eqs. $(15$, ,16), was extensively studied in 15, 44. For non-hindered transitions, the effect - the change of $\alpha_{i}^{0}$ - varied from negligible $\sim 10^{-2} \%$ (for low $\mathrm{Z}$ and all multipolarities except M1 and M2) to $10 \%$ (for high Z and multipolarity M1). For the hindered transitions, the situation is different. In [5], a lot of various transitions with the hindrance factors $F_{W}$ (related to the Weisskopf single particle estimate) from 10 up to $10^{16}$ were studied. It turned out, that the effect fluctuates between a few percent change up to a change by a factor of 20. Unfortunately, there is no universal connection between the hindrance factor and the magnitude of the effect. As an example, the $58 \mathrm{keV}$ E1 transition in ${ }_{72}^{180} \mathrm{Hf}$ has $F_{W}=10^{16}$ and the effect reaches the factor 2.5 [45. On the other hand, the $84 \mathrm{keV}$ E1 transition in ${ }_{91}^{231} \mathrm{~Pa}$ has $F_{W}=9 \times 10^{5}$ only and the ICC changes up to 20 times [46]. 
(Note that the change due to the intranuclear conversion is different for different atomic subshells. When present, the effect changes the ICC not only for the inner shells but also for the outer ones 47, 48.)

It is necessary to emphasize here that the intranuclear conversion itself brings no uncertainty into the theoretical ICC. The theoretical ICC are calculated using one of the above models 6, 41]. In many cases - when the effect of nuclear structure is small the theoretical ICC enabled the determination of nuclear characteristics (spins, parities) fairly well. However, when treating precise experimental data or hindered transitions, the intranuclear conversion must be taken into account. This means that the $I C C, \alpha_{i}$, in the form of Eqs.15,10) must be used instead of $\alpha_{i}^{0}$ and the parameters $\lambda, \eta, \xi$ should be fitted [43] into the experimental data. The parameters are determined together with their errors (standard deviations) but these errors are result of the fit and they do not affect the theoretical ICC $\alpha_{i}^{0}$.

Recommendation: If there is a discrepancy between the experimental and theoretical ICC and/or ICC ratios which cannot be explained by higher multipolarity admixture, always expect the intranuclear conversion and do fit the parameters $\lambda$ or $\xi$ and $\eta$.

\section{Danger of interpolation}

If there is no possibility to calculate ICC directly for the transition in question, one is forced to use ICC tables. The tables are also usually used for preliminary estimate of effects which can be expected in the detailed analysis. The tabulated ICC are always presented in some more or less dense energy mesh. When studying a particular transition, an interpolation is necessary.

It is generally believed that the dependence of ICC on the transition energy is close to linear one in the $\log -\log$ scale (i.e. $\log I C C \sim \log E_{t r}$ ). If so, the interpolation would make no problems. Unfortunately, in several cases the situation is not so simple (see e.g. graphs in [49]). For low-energy transitions a very non-monotonous dependence of ICC on energy was found in 50].

For the total ICC, the interpolation is strictly forbidden in the vicinity of the threshold of some atomic shell. As demonstrated in Fig. 3 there is a discontinuity in each point $E_{t r}=E_{b, i}$ where $E_{b, i}$ is the binding energy of some atomic subshell. The magnitude of this discontinuity depends on the transition multipolarity and on the particular subshell. (E.g. for $\mathrm{M} 1$, the discontinuity is greater for $s_{1 / 2}$ (i.e. $\mathrm{K}, \mathrm{L}_{1}, \mathrm{M}_{1}$ etc.) subshells and less for $p_{1 / 2}$ and $p_{3 / 2}$ subshells. For E2, it is just opposite.) Applying an interpolation in such a case would fetch in a very serious error. It is a pity that this error was not avoided in 49] (in graphs of total ICC, appendix F). 
Figure 3: Depencence of total ICC on transition energy for the E2 transition in element $\mathrm{Z}=126$. (Note that no discontinuity is observed at the $\mathrm{s}_{1 / 2}$-shell thresholds since the contribution of these shells into the total ICC is very small in this case.)

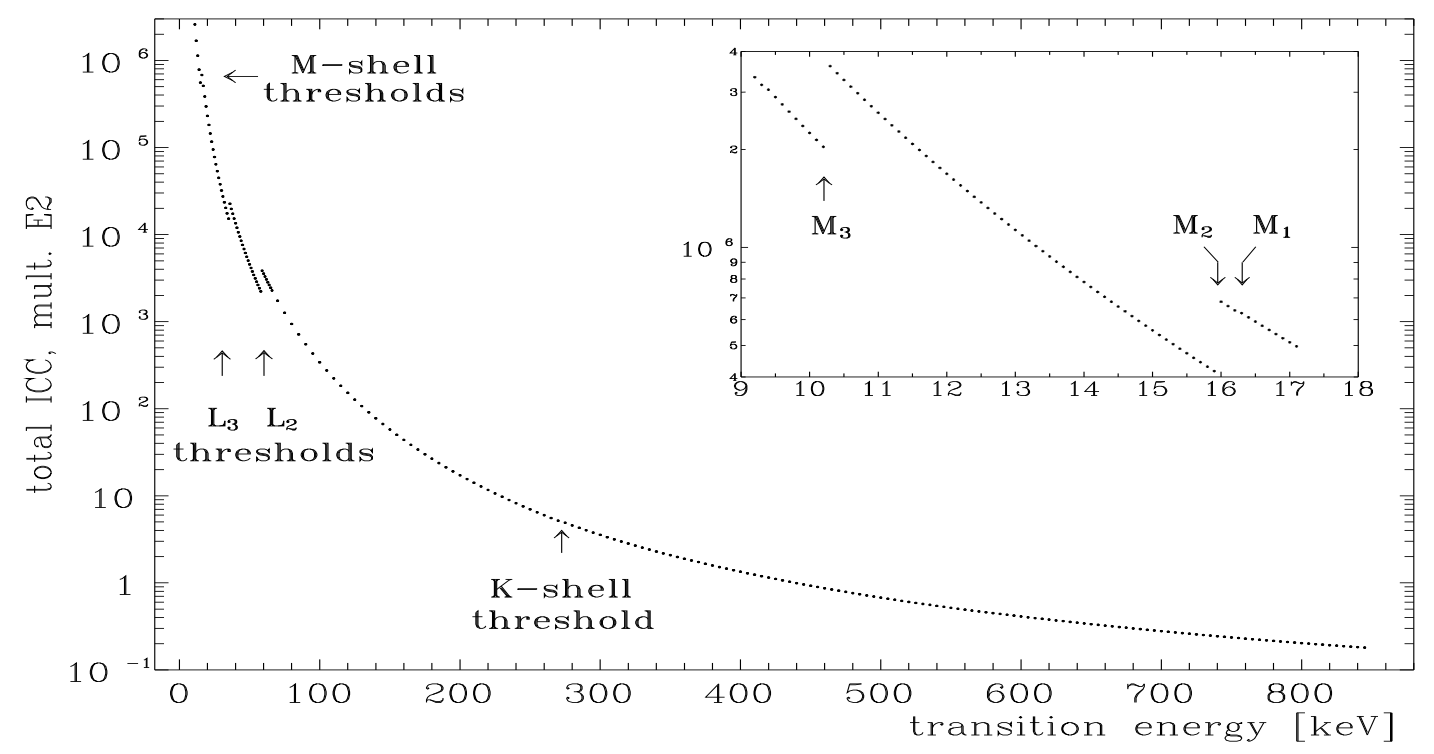

\section{Conclusions}

We have estimated the uncertainty of the theoretical ICC relevant to their most frequent application, i.e. the spin-parity determination of excited nuclear states. Until the uncertainties of the experimental ICC and/or their ratios are better than $10 \%$, any of the three tables [1, 2, 3] can be utilized for the comparison with the experiment and their uncertainties may be neglected.

When the accuracy of the experimental data is of a few percent, the uncertainty of the theoretical ICC as examined in this work should be included into the analysis which, in turn, leads to more realistic error of the final results (e.g. the multipole mixing parameters). As for the adding the uncertainties, we recommend to apply the quadratic addition as in the case of the statistical errors. We admit that the mentioned uncertainties are not of the pure statistical origin but the same argument as for the statistical errors can be used - it is improbable that all deviations assemble into the same direction.

To circumvent the question of errors caused by the atomic model which is not clear enough, the ICC for the cases of precise experimental data shoud be calculated directly for the particular transition energy using the HF atomic model (see Sect 3.1).

Keeping in mind the possibility of nuclear structure effect (Sect.3.7) in hindered transitions, more than one experimental quantity (ICC and/or ICC ratio) shoud be fetched into analysis whenever possible.

The interpolation in the tables of total ICC should be avoided. This is due to discontinuities of total ICC at the points of particular subshell's threshold. For lower transition energies, even the dependence of subshell ICC on the energy becomes nonmonotonous and the interpolation must be done with a great care. 
This work was supported by the Grant Agency of the Czech Republic under the contract No. 202/00/1625.

\section{References}

[1] F. Rösel, H.M. Fries, K. Alder, H.C. Pauli: At. Data Nucl. Data Tables 21 (1978) 91.

[2] I.M. Band, M.B. Trzhaskovskaya: Tables of the gamma-ray internal conversion coefficients for the K, L, M shells, $10 \leq \mathrm{Z} \leq 104$ (Leningrad: Nuclear Physics Institute, 1978).

[3] R.S. Hager, E.C. Seltzer: Nucl. Data A4 (1968) 1.

[4] M. Ryšavý, O. Dragoun: Tables of internal conversion coefficients for superheavy elements, Report NPI Řž-TH-01/2000 (http://xxx.lanl.gov/e-print/nucl-th/0004003).

[5] I.M. Band, M.A. Listengarten, A.P. Feresin: Anomalies in the gamma-ray internal conversion coefficients (Nauka, Leningrad 1976), in Russian.

[6] M.E. Rose: Internal conversion coefficients, North-Holland, Amsterdam 1958.

[7] L.A. Sliv, I.M. Band: Tables of gamma-ray internal conversion coefficients, part I. K-shell, Izdat. AN SSSR, Moskva-Leningrad 1956 (in Russian).

[8] L.A. Sliv, I.M. Band: Tables of gamma-ray internal conversion coefficients, part II. - L-shell, Izdat. AN SSSR, Moskva-Leningrad 1958 (in Russian).

[9] O. Dragoun, H.C. Pauli, F. Schmutzler: Nucl. Data Tables A6 (1969) 235.

[10] O. Dragoun, V. Brabec, M. Ryšavý, A. Špalek: Z. Physik A281 (1977) 347.

[11] O. Dragoun, M. Ryšavý, F. Bečvář, V. Brabec: Czech. J. Phys. 31 (1981) 246.

[12] I.M. Band, M.A. Listengarten, M.B. Trzhaskovskaya: Izv. AN SSSR, ser. fiz., 45 (1981) 829; V.O. Sergeev, M.A. Listengarten: ibid. p.814; M.A. Listengarten, V.O. Sergeev: in: Strong and weak arguments in nuclear spectroscopy and nuclear theory, ed. B.S. Dzhelepov, Nauka, Leningrad 1981, p. 39 (in Russian).

[13] V.A. Zilitis, V.F. Trusov, M.O. Eglajs: Izv. AN SSSR, ser. fiz., 45 (1981) 690;

[14] I.M. Band, M.A. Listengarten, M.B. Trzhaskovskaya: Izv. AN SSSR, ser. fiz., 53 (1989) 910; I.M. Band, M.A. Listengarten, M.B. Trzhaskovskaya: Izv. AN SSSR, ser. fiz., 54 (1990) 15; I.M. Band, M.B. Trzhaskovskaya: At. Data Nucl. Data Tables 55 (1993) 43. 
[15] M.A. Coulthard: Proc. Phys. Soc. (London) 91 (1967) 44; Report UMP-66/4, Un. of Melbourne, Victoria, 1966.

[16] C. Caso et al. (Particle Data Group): Eur. Phys. J. C3 (1998) 1.

[17] J.J. Matese: Phys. Rev. 173 (1968) 165.

[18] L.A. Borisoglebskii, B.I. Tesevich, G.S. Shulyakovskii: Izv. AN SSSR, ser. fiz., 38 (1974) 2581.

[19] E.L. Church, J. Weneser: Ann. Rev. Nucl. Sci 10 (1960) 193.

[20] V.A. Krutov: Izv. AN SSSR, ser. fiz., 22 (1958) 162; V.A. Krutov, K. Myuller: ibid., p. 171.

[21] V.A. Krutov, V.N. Fomenko: Ann. der Phys. 21 (1968) 291; Izv. AN SSSR, ser. fiz., 33 (1969) 84.

[22] V.A. Krutov, I.M. Knyazkov: Ann. der Phys. 25 (1970) 10; Izv. AN SSSR, ser. fiz., 36 (1972) 2190.

[23] V.A. Krutov: Pis'ma ZhETF 52 (1990) 1176.

[24] R. Hager, E. Seltzer: Phys. Rev. C2 (1970) 902.

[25] H.C. Pauli, K. Alder, R.M. Steffen: in: The electromagnetic interactions in nuclear spectroscopy, ed. W.D. Hamilton, North-Holland, Amsterdam 1975, p. 341.

[26] U. Raff, H.C. Pauli: Z. Physik A275 (1972) 145.

[27] R. Mayol, J.D. Martinez, F. Salvat, J. Parellada: Z. Phys. A - Atoms and Nuclei 316 (1984) 251.

[28] D. Hinneburg, M. Nagel: Symposium "Wechselwirkung ionisirender Strahlung", Gera, Sept. 1977.

[29] D. Hinneburg, M. Nagel, G.Z. Brunner: Z. Physik A291 (1979) 13.

[30] D. Hinneburg: Z. Physik A300 (1981) 129.

[31] S. Gasiorowicz: Elementary particle physics, J. Wiley \& Sons, New York, 1966.

[32] K.D. Sevier: At. Data Nucl. Data Tables 24 (1974) 323.

[33] C.C. Lu, T.A. Carlson, F.B. Malik, T.C. Tucker, C.W. Nestor, Jr.: At. Data 3 (1971) 1.

[34] I.M. Band, M.A. Listengarten, M.B. Trzhaskovskaya: in: Precise measurements in nuclear spectroscopy, Mokslas, Vilnius 1984, p. 50 (in Russian).

[35] E. Hartmann, M. Ryšavý: Hyp. Interactions 13 (1983) 257. 
[36] V. Brabec, M. Ryšavý, O. Dragoun, M. Fišer, A. Kovalík, Cs. Ujhelyi, D. Berényi: Z. Physik A306 (1982) 347.

[37] M. Ryšavý, O. Dragoun: Europhys. Lett. 17 (1992) 303.

[38] M. Fujioka, M. Takashima, M. Kanbe, O. Dragoun, M. Ryšavý: Z. Physik A299 (1981) 283.

[39] M.M. Vsevolodov, D.P. Grechukhin, A.A. Soldatov: Izv. AN SSSR, ser. fiz., 54 (1990) 58.

[40] T.A. Green, M.E. Rose: Phys. Rev 110 (1958) 105.

[41] L.A. Sliv: Zhur. Exp. Teor. Fiz. 21 (1951) 770.

[42] H.C. Pauli: Helv. Phys. Acta 40 (1967) 713.

[43] M. Ryšavý, O. Dragoun: Comput. Phys. Commun. 19 (1980) 93.

[44] M.A. Listengarten: Izv. AN SSSR, ser. fiz., 42 (1978) 1823.

[45] G. Scharff-Goldhaber, M. McKeown: Phys. Rev 158 (1967) 105.

[46] F. Asaro, F.S. Stephens, J.M. Hollander, I. Perlman: Phys. Rev. 17 (1960) 492.

[47] O. Dragoun, Z. Plajner, B. Martin, A. v. Kap-Herr: Phys. Lett. 29B (1969) 221.

[48] O. Dragoun, H. Blumberg: Czech. J. Phys. B21 (1971) 785.

[49] R.B. Firestone, C.M. Baglin, S.Y. Frank Chu: Table of Isotopes, 8th edition, J. Wiley \& Sons, New York, 1998.

[50] O. Dragoun, M. Ryšavý, C. Günther: Phys. Rev. 47 (1993) 870. 